\title{
Electromagnetic Field and Three-Phase-Lag in a Compressed Rotating Isotropic Homogeneous Micropolar Thermo-Viscoelastic Half-Space
}

\author{
S.M. Abo-Dahab ${ }^{1}$, A. Abd-Alla ${ }^{2}$, and araby kilany ${ }^{3}$ \\ ${ }^{1} 1$ Math. Dept., Faculty of Science, South Valley University, Qena 83523, Egypt \\ ${ }^{2}$ Faculty of Science, Taif University 888, Saudi Arabia \\ ${ }^{3}$ Sohag University
}

November 19, 2020

\begin{abstract}
A unified mathematical model of three-phase-lag in a compressed rotating isotropic homogeneous micropolar thermo-viscoelastic medium based on a ramp type thermal shock is developed. An application of this model is carried out to resolve the problem of a perfectly conducting half-space subjected to certain boundary conditions in the presence of an electromagnetic field. Lame's potentials and normal mode analysis techniques are employed to get the general analytical solutions. Specific attention is paid to explore the impact of the rotation, magnetic field, ramp time, as well as initial stress on the distributions of temperature, displacement, stress, and induced electric and magnetic distribution. The findings show that the impact of the rotation, magnetic field, viscous, ramp parameter, initial stress, and phase-lag on the micropolar thermo-viscoelastic medium is noticeable.
\end{abstract}

\section{Hosted file}

Paper to MMA-20-19780.pdf available at https://authorea.com/users/292909/articles/ 493921-electromagnetic-field-and-three-phase-lag-in-a-compressed-rotating-isotropichomogeneous-micropolar-thermo-viscoelastic-half-space 